\title{
The effects of host-feeding on stability of discrete-time host-parasitoid population dynamic models
}

\author{
Brooks Emerick $^{\mathrm{a}}$, Abhyudai Singh ${ }^{\mathrm{b}, \mathrm{c}}$ \\ ${ }^{a}$ Department of Mathematics, Trinity College, Hartford CT, 06106 \\ ${ }^{b}$ Department of Electrical \& Computer Engineering, University of Delaware, Newark DE, \\ 19716 \\ ${ }^{c}$ Department of Biomedical Engineering, University of Delaware, Newark DE, 19716
}

\begin{abstract}
Discrete-time models are the traditional approach for capturing population dynamics of a host-parasitoid system. Recent work has introduced a semidiscrete framework for obtaining model update functions that connect hostparasitoid population levels from year-to-year. In particular, this framework uses differential equations to describe the host-parasitoid interaction during the time of year when they come in contact, allowing specific behaviors to be mechanistically incorporated. We use the semi-discrete approach to study the effects of host-feeding, which occurs when a parasitoid consumes a potential host larva without ovipositing. We find that host-feeding by itself cannot stabilize the system, and both populations exhibit behavior similar to the Nicholson-Bailey model. However, when combined with stabilizing mechanisms such as density-dependent host mortality, host-feeding contracts the region of parameter space that allows for a stable host-parasitoid equilibrium. In contrast, when combined with a density-dependent parasitoid attack rate, host-feeding expands the non-zero equilibrium stability region. Our results show that host-feeding causes inefficiency in the parasitoid population, which yields a higher population of hosts per generation. This suggests that hostfeeding may have limited long-term impact in terms of suppressing host levels for biological control applications.
\end{abstract}

Keywords: host-parasitoid, host-feeding, semi-discrete model, Nicholson-Bailey, functional response

Email addresses: brooks.emerick@trincoll.edu (Brooks Emerick), absingh@udel.edu (Abhyudai Singh)

Preprint submitted to Mathematical Biosciences

November 13, 2015

(C) 2015. This manuscript version is made available under the Elsevier user license http://www.elsevier.com/open-access/userlicense/1.0/ 


\section{Introduction}

The host-parasitoid dynamic typically involves a vulnerable period during the year in which hosts are susceptible to attack by parasitoids. There exists a tendency of synovigenic parasitoids to eat the host without laying eggs inside $(1 ; 2)$. Generally, an adult female parasitoid emerges every year with less eggs than she can potentially oviposit in her lifespan. Therefore, a parasitoid will feed on hosts to gain the necessary energy and tissue to mature additional eggs $(3 ; 4)$. However, in doing so, the parasitoid loses a potential host as the majority of parasitoids kill the host during host-feeding. This results in a high death rate of the host population during the vulnerable period, which can have interesting consequences on later generations $(5 ; 6)$. For further reviews on the host-feeding interaction and its biological implications, we refer the interested reader to $(3 ; 7 ; 8)$.

A traditional approach to describe parasitoid-host dynamics is to use discrete time models, such as the Nicholson-Bailey model (9) and others considered more recently $(10 ; 11 ; 12 ; 13 ; 14)$. Discrete models can monitor the change in population density for distinct points in time, such as each year or each generation, which agrees with typical life-cycle themes of insects in temperate climates (15). Several authors incorporate the effects of hostfeeding in continuous models, which are suitable to year-round interactions such as those in tropical climates. They conclude that host-feeding can have stabilizing effects $(16 ; 17)$ or no effect on stability $(18 ; 19 ; 20)$, while Shea et. al. conclude that egg production delay has a destabilizing effect in hostfeeding models (21). Further reviews on past and recent models are provided by Hassell in (22) and (23) and by Murdoch et. al. in (15).

In this work, we use the semi-discrete framework (24) to develop discrete update functions based on a continuous host-feeding dynamic. This modeling approach is more mechanistic as compared to early phenomenological models because it incorporates specific host-parasitoid interactions during the vulnerable period. We conclude that host-feeding has no effect on stability in contrast to previous results by Singh and Nisbet (25); however, host-feeding combined with density-dependent mortality rates can have both a stabilizing and destabilizing effect. When combined with a quadratic functional response, host-feeding stabilizes the system. The paper is organized as follows: we formulate the model in Section 2; in Section 3, we consider 
the simplest case and show that host-feeding alone does not stabilize the Nicholson-Bailey model (9); in Sections 4 and 5, we incorporate the effects of density-dependent mortality and quadratic functional response, respectively, and consider the stability of the system; in Section 6, we discuss the effect of egg maturation delay on the quadratic functional response model; and, we conclude with a discussion in Section 7.

\section{Model Formulation}

Generally, the discrete-time model used to describe the host-parasitoid dynamics is given by

$$
\begin{aligned}
H_{t+1} & =F\left(H_{t}, P_{t}\right) \\
P_{t+1} & =G\left(H_{t}, P_{t}\right),
\end{aligned}
$$

where $H_{t}$ and $P_{t}$ are the adult female host and parasitoid densities, respectively, at the beginning of the vulnerable period of year $t$, where $t$ is an integer. In this model, we track only female hosts and parasitoids. Figure 1 illustrates the life cycle of the host and parasitoids in a year long period. During year $t$, host pupae mature into $H_{t}$ adults. These $H_{t}$ female adults lay

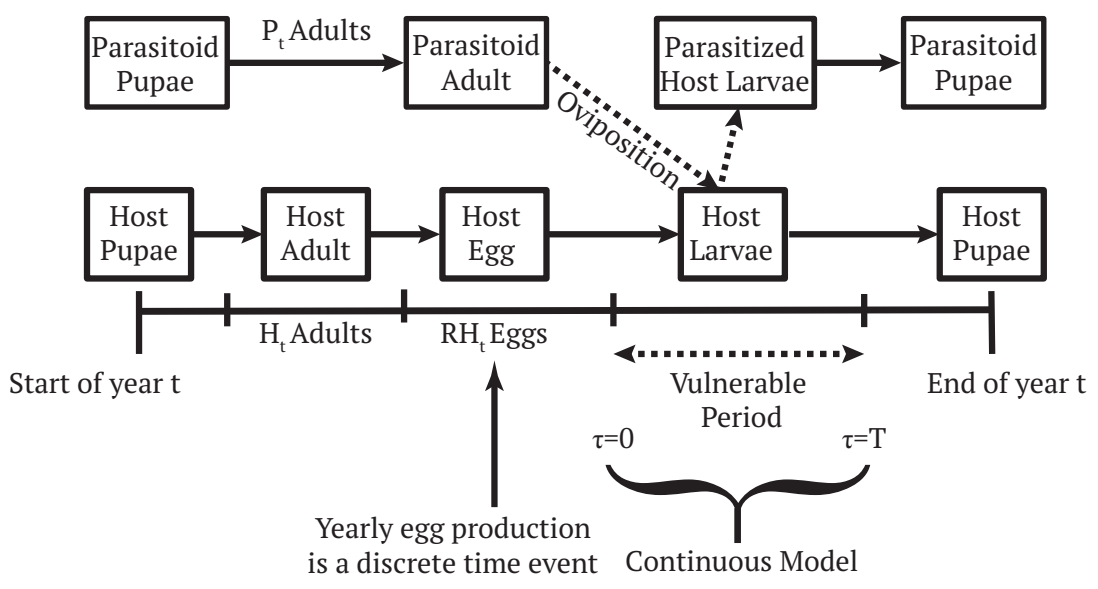

Figure 1: Life cycle of the host and parasitoids in year $t$ with a description of the modeling approach. 
a particular amount of eggs, which eventually mature into $R H_{t}$ larvae at the beginning of the vulnerable stage. Here, $R>1$ denotes the number of viable eggs produced by each adult host. The time within the vulnerable stage is denoted by $\tau$, where $\tau \in[0, T]$. Time $\tau=0$ denotes the beginning of the vulnerable stage and $\tau=T$ denotes the end. During this time, adult female parasitoids emerge with eggs and seek out host larvae to oviposit inside. The parasitoid egg inside the host becomes a juvenile parasitoid that uses the host as a resource. At the end of the vulnerable period, a proportion of the host population is infected. This results in an initial parasitoid population the next year as juvenile parasitoids emerge from the host to continue their life cycle. A number of hosts escape parasitism, and survive to mature into pupae, which pupate over the winter creating the next year's population of adult hosts. To better understand the discrete update of each year, we consider a continuous model to describe the interaction of hosts and parasitoids during the vulnerable period. Next, we discuss the approach to model this system of interactions.

\subsection{The Semi-Discrete Framework}

A continuous time model is used to describe the dynamics of the interacting host and parasitoid populations during the vulnerable period. The update functions $F$ and $G$ of the discrete model depend on the output of the continuous model at the end of the vulnerable period each year. We consider the following chemical reaction

$$
P+L \stackrel{g(\cdot)}{\longrightarrow} I+P \text {. }
$$

Here, $g(\cdot)$ (units: time $^{-1}$ parasitoid ${ }^{-1}$ ) is the attack rate of the parasitoids, which represents the instantaneous rate at which the hosts are attacked per parasitoid. This function could potentially be dependent on the population of hosts, parasitoids, or infected hosts. In general, we write the continuous model as the following system of ordinary differential equations in the time variable $\tau$ :

$$
\begin{aligned}
& \frac{d L(\tau, t)}{d \tau}=-g(\cdot) L(\tau, t) P(\tau, t) \\
& \frac{d I(\tau, t)}{d \tau}=g(\cdot) L(\tau, t) P(\tau, t) \\
& \frac{d P(\tau, t)}{d \tau}=0 .
\end{aligned}
$$


where $L(\tau, t), I(\tau, t)$, and $P(\tau, t)$ denote the concentrations of uninfected host larvae, infected host larvae, and parasitoids, respectively, at time $\tau$ during the vulnerable period in year $t$. We note that this system assumes no host or parasitoid mortality as well as no egg limitation in the parasitoids. We subject the system to the following initial conditions

$$
L(0, t)=R H_{t}, \quad I(0, t)=0, \quad P(0, t)=P_{t} .
$$

These equations are integrated from $\tau=0$ to $\tau=T$. Assuming each parasitized host larvae gives rise to $k$ adult parasitoids in the next generation, the update functions in the discrete model are

$$
\begin{aligned}
H_{t+1} & =F\left(H_{t}, P_{t}\right):=L(T, t) \\
P_{t+1} & =G\left(H_{t}, P_{t}\right):=k I(T, t) .
\end{aligned}
$$

Here, the parameter $k$ is the number of parasitoid larvae that emerge from one infected host larva, which may be larger than one depending on how many eggs are oviposited. Once the system is formulated, investigation into the stability region of Equations (1) and (2) can be carried out using the standard Jury conditions (26). If we let $g=c$, i.e. a constant parasitoid attack rate, then we obtain the following update

$$
\begin{aligned}
F\left(H_{t}, P_{t}\right) & =R H_{t} e^{-c P_{t} T} \\
G\left(H_{t}, P_{t}\right) & =k R H_{t}\left(1-e^{-c P_{t} T}\right),
\end{aligned}
$$

which is the classic Nicholson-Bailey model. This interaction is unstable and over time both populations experience diverging oscillations (15). Several changes in the form of functional responses, susceptibility of attack, and density-dependent mortality can be made to stabilize the system as in $(25 ; 27)$. Next, we illustrate how we incorporate host-feeding into the semidiscrete framework and discuss how to obtain the update functions.

\subsection{The Semi-Discrete Framework with Host-Feeding}

The continuous model we consider includes the tendency of the parasitoids to feed on host larvae without infecting them with an egg $(3 ; 4)$. This means the parasitoid population has two phases: without egg and with egg. If a parasitoid lays an egg inside the larva, an infected larva is produced and the parasitoid is now without an egg. This eggless parasitoid must feed on a host larva before gaining another egg; however, if a parasitoid has an egg, 
it will infect the host larva rather than feed. Implicit to our model is the assumption that once a parasitoid feeds on the host, it immediately gains enough energy to produce an egg, i.e., the transitional period from eggless to with egg is instantaneous; however, we do discuss consequences of a delay in Section 6. This approach is motivated by the work of Shea et. al. (21), who consider several parasitoid phases with an egg maturation delay. A kinetic diagram below describes the process,

$$
\begin{aligned}
& P_{1}+L \stackrel{g(\cdot)}{\longrightarrow} I+P_{0} \\
& P_{0}+L \stackrel{g(\cdot)}{\longrightarrow} P_{1} .
\end{aligned}
$$

Here, the function $g(\cdot)$ (units: time $^{-1}$ parasitoid $^{-1}$ ) represents two means by which the parasitoids attack hosts. The first reaction is the instantaneous rate at which the hosts are parasitized or infected by a mature parasitoid. The second reaction represents the rate at which the hosts are devoured by an eggless parasitoid. We assume the two rates are equal as this leads to analytic results. In the most general case, both rates may potentially depend on the host, parasitoid, or infected host populations, which yields the following continuous model

$$
\begin{aligned}
\frac{d L(\tau, t)}{d \tau} & =-g(\cdot)\left(P_{0}(\tau, t)+P_{1}(\tau, t)\right) L(\tau, t) \\
\frac{d I(\tau, t)}{d \tau} & =g(\cdot) P_{1}(\tau, t) L(\tau, t) \\
\frac{d P_{0}(\tau, t)}{d \tau} & =g(\cdot)\left(P_{1}(\tau, t)-P_{0}(\tau, t)\right) L(\tau, t) \\
\frac{d P_{1}(\tau, t)}{d \tau} & =-g(\cdot)\left(P_{1}(\tau, t)-P_{0}(\tau, t)\right) L(\tau, t),
\end{aligned}
$$

where $L(\tau, t), I(\tau, t), P_{0}(\tau, t)$, and $P_{1}(\tau, t)$ are the density of uninfected host larvae; parasitized host larvae; eggless parasitoids; and parasitoids with egg, respectively, at time $\tau$ during the vulnerable period in year $t$. We subject the system to the following initial conditions

$$
L(0, t)=R H_{t}, \quad I(0, t)=0, \quad P_{0}(0, t)=\lambda P_{t}, \quad P_{1}(0, t)=(1-\lambda) P_{t} .
$$

This initial condition assumes that a proportion of the parasitoid population emerges eggless. We consider this case because psychological and behavioral factors including resorption and nutrition may contribute to the specific egg 
load at any given time (28). We discuss the results of this assumption briefly in each section and we see that for $\lambda=1 / 2$, the model is remarkably similar to that of Singh and Nisbet's without host-feeding (25). These equations are integrated from $\tau=0$ to $\tau=T$. Assuming each parasitized host larva gives rise to $k$ adult parasitoids in the next generation, the yearly update functions are the same as before in Equations (8) and (9). We note here that total parasitoid density is fixed, i.e. $P_{t}=P_{0}(\tau, t)+P_{1}(\tau, t)$ since there is no parasitoid mortality of any kind. There is no larval mortality as well except that due to parasitization from oviposition and host-feeding. Also, we note that this model assumes parasitoids only feed on uninfected host larvae as it is reasonable for a parasitoid to be able to detect infection. For the remainder of the paper, we suppress the dependence on $\tau$ and $t$ for the functions $L, I, P_{0}$, and $P_{1}$ for convenience.

\section{Nicholson-Bailey with Host-Feeding}

In this section, we consider the most simple case. We assume the attack rate $g(\cdot)$ in Equations (12) and (13) is constant, i.e. $g=c$. Our continuous time model during the vulnerable period from Equations (14)-(17) becomes

$$
\begin{aligned}
\frac{d L}{d \tau} & =-c\left(P_{0}+P_{1}\right) L \\
\frac{d I}{d \tau} & =c P_{1} L \\
\frac{d P_{0}}{d \tau} & =c\left(P_{1}-P_{0}\right) L \\
\frac{d P_{1}}{d \tau} & =-c\left(P_{1}-P_{0}\right) L .
\end{aligned}
$$

We can solve the system for $L$ and $I$ explicitly (Appendix A) and our discrete update from Equations (1) and (2) can be written as

$$
\begin{aligned}
H_{t+1} & =R H_{t} e^{-c P_{t} T} \\
P_{t+1} & =\frac{k R H_{t}}{2}\left(1-e^{-c P_{t} T}\right)+\left(\frac{1}{2}-\lambda\right) k P_{t}\left\{\exp \left[\frac{R H_{t}}{P_{t}}\left(1-e^{-c P_{t} T}\right)\right]-1\right\} .
\end{aligned}
$$

The fixed points to this model satisfy

$$
H^{*}=\frac{\beta \ln R}{c T(R-1)}, \quad P^{*}=\frac{\ln R}{c T},
$$


where $\beta=\beta(\lambda, k)$ is given by the following function:

$$
\beta(\lambda, k)=1-2 \lambda+\frac{2}{k}-x^{*},
$$

where $x^{*}$ is a solution to the equation

$$
x e^{x}=(1-2 \lambda) \exp \left(1-2 \lambda+\frac{2}{k}\right) .
$$

This equation originates from the LambertW function (also called the omega function). Further details of how $\beta$ depends on $\lambda$ and $k$ are contained in Appendix A. In the special case that $\lambda=1 / 2$ (equal proportion of eggless and with egg parasitoids at the start of the vulnerable period), our update functions reduce to

$$
\begin{aligned}
H_{t+1} & =R H_{t} e^{-c P_{t} T} \\
P_{t+1} & =\frac{k R H_{t}}{2}\left(1-e^{-c P_{t} T}\right),
\end{aligned}
$$

which is identical to the Nicholson-Bailey model of Equations (10)-(11) except for a factor of $1 / 2$ in the equation for $P_{t+1}$. Here, $x^{*}=0$ so that $\beta=2 / k$ and the fixed point for the host population is

$$
H^{*}=\frac{2 \ln R}{c k T(R-1)},
$$

which is exactly double that of the Nicholson-Bailey model. This makes sense as only half the parasitoid population are ovipositing at the beginning of every year, which interestingly results in a doubling of the host population. For $\lambda \neq 1 / 2$, we consider the dynamics with $k=1$, since our model assumes that parasitoids only carry a single egg and thus only a single parasitoid can emerge from an infected host. In this case, we see that $\beta(1, \lambda)=\beta(\lambda)$ is a increasing function of $\lambda$, indicating that the equilibrium host population density increases as the proportion of initially eggless parasitoids increases. Also, (without loss of generality and for the remainder of the paper) we let $T=1$, which corresponds to one vulnerable period (approximately 90 days) as this parameter typically only has a scaling effect on the stability region. (A similar effect is observed with the parameter $k$.) We are interested in the case when $\lambda=0$ as this implies that all parasitoids emerge with egg, which is 
similar to Singh and Nisbet's semi-discrete model without host-feeding (25). As shown in Appendix A, this model is unstable for $R>1$. In fact, when $\lambda=0$, we have $\beta=0.7921$, indicating that a portion of the host population is lost at equilibrium. This suggests that host-feeding has a slight destabilizing effect, in the sense that oscillations grow faster from the equilibrium point, on the already unstable Nicholoson-Bailey model. Therefore, host-feeding alone cannot establish stability, which is in agreement to previous models $(18 ; 19)$.

\section{Density-Dependent Mortality with Host-Feeding}

In this section, we consider the effects of host-feeding when there is a density-dependent mortality in the host or parasitoid population. We find that the host-feeding dynamic destabilizes the host mortality model but there is numerical evidence to suggest that host-feeding stabilizes the model when a mortality is included in the parasitoid dynamics.

\subsection{Density-Dependent Host Mortality}

It is shown in both phenomenological models $(29 ; 30)$ and mechanistic approaches (25) that density-dependent host mortality can stabilize discretetime systems. To investigate stability with host-feeding, we implement a density-dependent host mortality in Equations (14)-(17). The kinetic reactions of the system are depicted as

$$
\begin{aligned}
& P_{1}+L \stackrel{g(\cdot)}{\longrightarrow} I+P_{0} \\
& P_{0}+L \stackrel{g(\cdot)}{\longrightarrow} P_{1} \\
& L \stackrel{g_{1}(\cdot)}{\longrightarrow} \text { Death. }
\end{aligned}
$$

The function $g_{1}(\cdot)$ (units: time $^{-1}$ ) represents the host mortality rate due to causes other than parasitism and host-feeding. We assume the attack rates are constant, $g=c$, implying a linear functional response. We let the host mortality rate depend on the current amount of host larvae, i.e., $g_{1}=$ $c_{1} L$ so that density-dependent effects can act simultaneously with parasitism in the continuous-time model. We also assume that the parasitized larvae immediately become juvenile parasitoids and do not compete for resources with the host larvae, and hence, do not have a density-dependent mortality. 
We obtain the following system for $\tau \in[0, T]$,

$$
\begin{aligned}
\frac{d L}{d \tau} & =-c\left(P_{1}+P_{0}\right) L-c_{1} L^{2} \\
\frac{d I}{d \tau} & =c P_{1} L \\
\frac{d P_{0}}{d \tau} & =c\left(P_{1}-P_{0}\right) L \\
\frac{d P_{1}}{d \tau} & =-c\left(P_{1}-P_{0}\right) L
\end{aligned}
$$

subject to the same initial conditions as above. Solving this system explicitly (Appendix B) yields the following discrete update system

$$
\begin{aligned}
H_{t+1} & =\frac{R H_{t} e^{-c P_{t} T}}{f\left(H_{t}, P_{t}\right)} \\
P_{t+1} & =\frac{c k P_{t}}{2 c_{1}} \ln \left[f\left(H_{t}, P_{t}\right)\right]+\left(\lambda-\frac{1}{2}\right) \frac{k P_{t}}{2}\left[f\left(H_{t}, P_{t}\right)^{-\frac{2 c}{c_{1}}}-1\right],
\end{aligned}
$$

where

$$
f\left(H_{t}, P_{t}\right)=1+c_{1} R H_{t} \frac{1-e^{-c P_{t} T}}{c P_{t}} .
$$

We obtain two nontrivial fixed point solutions. The no-parasitoid (NP) fixed point equilibrium is given by

$$
H_{N P}^{*}=\frac{R-1}{c_{1} T R}, \quad P^{*}=0 .
$$

The stability analysis in Appendix B shows that the no-parasitoid equilibrium is stable for

$$
\frac{\ln R}{\gamma}<\frac{c_{1}}{c}
$$

where $\gamma=\gamma(\lambda, k)$ is given by the following function:

$$
\gamma(\lambda, k)=\lambda-\frac{1}{2}+\frac{2}{k}+\frac{y^{*}}{2}
$$

where $y^{*}$ is a solution to the equation

$$
y e^{y}=(1-2 \lambda) \exp \left(1-2 \lambda-\frac{4}{k}\right) .
$$


Similar to the Nicholson-Bailey case, this equation originates from the LambertW function. Further details of how $\gamma$ depends on $\lambda$ and $k$ are contained in Appendix B. In the simple case when $\lambda=1 / 2$, we have $y^{*}=0$ so that $\gamma=2 / k$, which means the NP fixed point is stable for

$$
\frac{\ln R}{2}<\frac{c_{1}}{k c} .
$$

In this case, our model is almost identical to Singh and Nisbet's model without host-feeding (25). In fact, we obtain the same NP fixed point but the region of stability is doubled due to host-feeding (see Figure 2). To

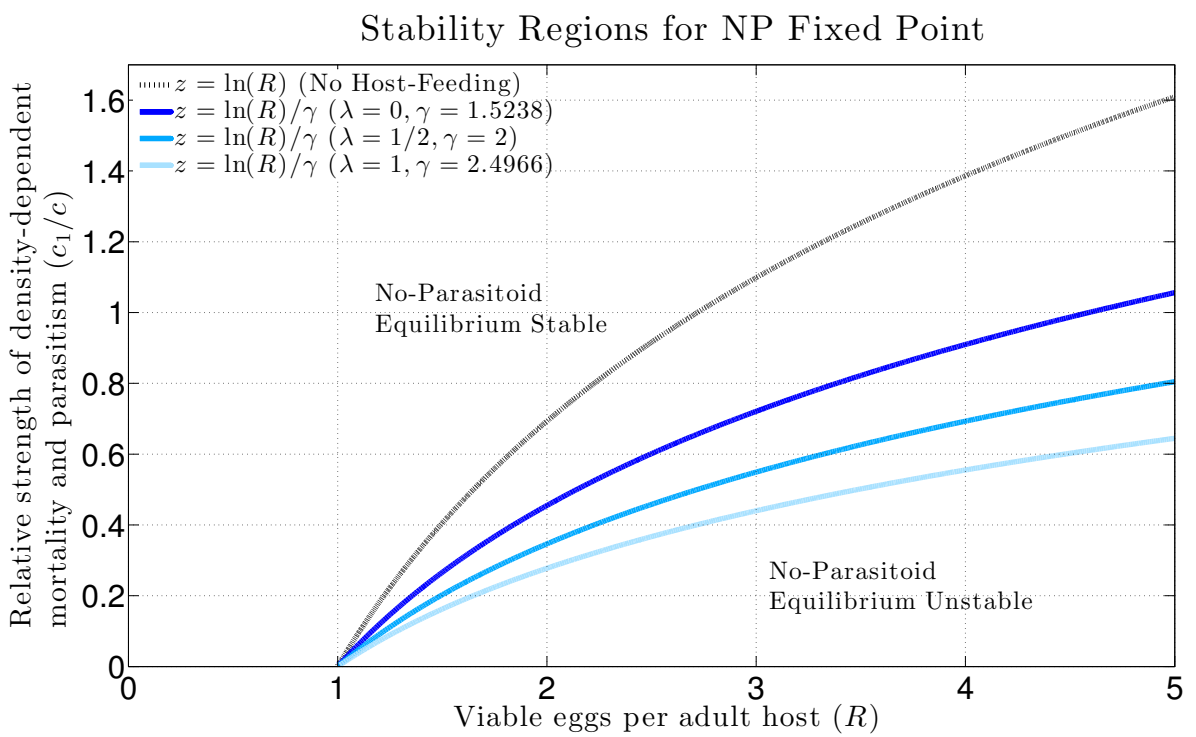

Figure 2: Host-Feeding increases the no-parasitoid equilibrium stability region of the Nicholson-Bailey model with density-dependent host mortality. The stability region specified in Equation (38) for the discrete time, host mortality model (Equations (35) and (36)) as a function of the strength of density-dependent mortality to parasitism $\left(c_{1} / c\right)$ and the number of viable eggs per host, $R$. The stability region is larger with hostfeeding as compared to the density-dependent host mortality model without host-feeding (dotted black line). In fact, as $\lambda$ (the proportion of initially eggless parasitoids each year) increases, the stability region increases (solid blue lines). Parameters: $\lambda=0$ (dark blue), $\lambda=1 / 2$ (blue), $\lambda=1$ (light blue), $k=1$, and $T=1$. 
determine the effect of host-feeding on the NP fixed point, we let $\lambda=0$ and $k=1$, since this corresponds to ideal conditions for oviposition at the beginning of the vulnerable period. We find that $\gamma=1.5238$, which indicates that host-feeding doesn't restrict the NP fixed point stability region to that of the model without host-feeding. In Figure 2 , as $\lambda \rightarrow 0$, we see that the NP fixed point stability region is minimized, but it never gets as small as that of the model without host-feeding. Therefore, we may conclude that the host-feeding dynamic creates a burden on the parasitoid expansion since potential host larvae are devoured rather than used for reproduction.

We note that there is no NP equilibrium in the previous model because there is no density-dependent mortality to limit the host population for small concentrations of parasitoids. When there is a host mortality, a noparasitoid fixed point emerges and for sufficiently large values of $c_{1} / c$, which describes the strength of density-dependent mortality versus parasitism, this fixed point is stable.

The second equilibrium point characterizes a presence of both hosts and parasitoids, and is given by

$$
H^{*}=\left(\frac{\exp \left(\frac{\gamma c_{1}}{c}\right)-1}{R-\exp \left(\frac{\gamma c_{1}}{c}\right)}\right) \frac{c P^{*}}{c_{1}}, \quad P^{*}=\frac{\ln (R)-\frac{\gamma c_{1}}{c}}{c T} .
$$

where $\gamma=\gamma(\lambda, k)$ is defined in Equation (39). We consider the region of stable solutions for various values of $c_{1} / c$. We seek the stability region when $\lambda=0$ and $k=1$ as this corresponds to all parasitoids emerging with a single egg at the beginning of the vulnerable. With this parameter set, we are able to compare directly to the Singh and Nisbet model without host-feeding. An analysis in Appendix B shows the equilibrium point is stable for

$$
z^{*}<\frac{c_{1}}{c}<\frac{\ln R}{\gamma}
$$

where $z^{*}$ satisfies the following equation

$$
\begin{gathered}
\frac{\left\{R\left[\left(\gamma z^{*}+1\right)-\ln R\right]\left(1-e^{-\gamma z^{*}}\right)+1-e^{\gamma z^{*}}\right\}\left(1+e^{-2 \gamma}\right)}{2 z^{*}\left(e^{\gamma z^{*}}-R\right)}+\cdots \\
\frac{1}{2}\left[\gamma+\frac{1}{2}\left(1-e^{-2 \gamma}\right)\right] e^{-\gamma z^{*}}=1 .
\end{gathered}
$$

and $\gamma=\gamma(0,1)=1.5238$. Figure 3 shows the stability region as compared to 
Stability Regions for Non-Zero Fixed Point

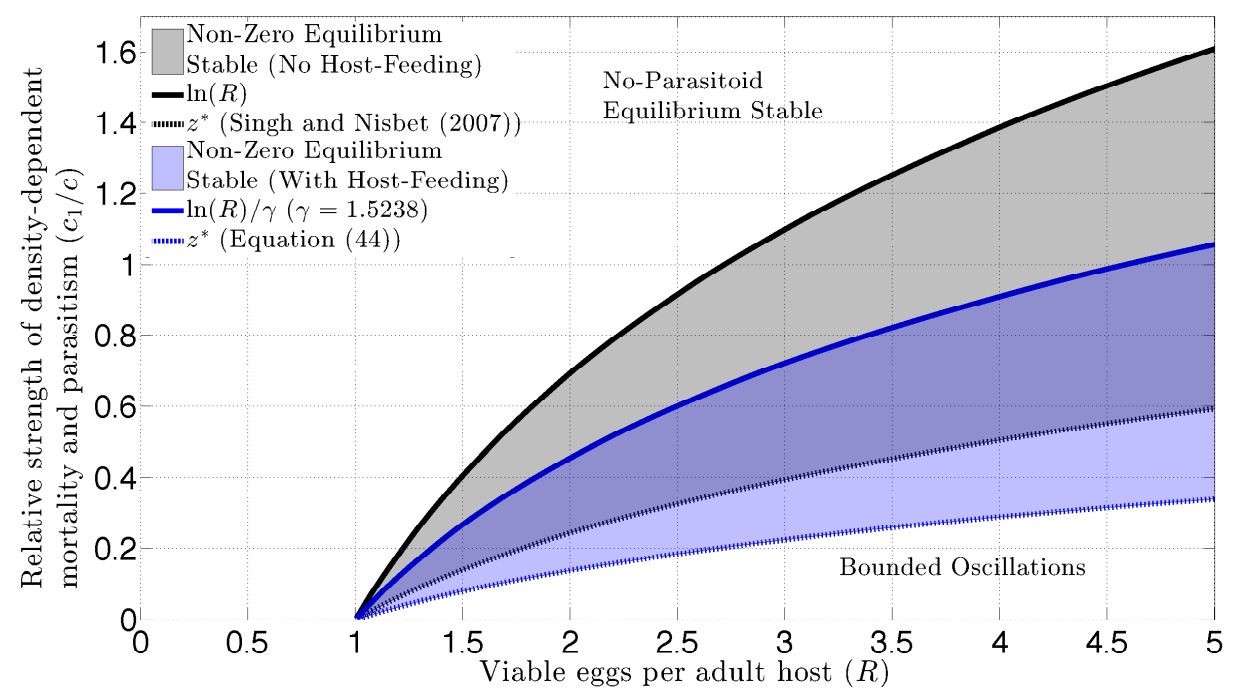

Figure 3: Host-feeding decreases the host-parasitoid equilibrium stability region of the Nicholson-Bailey model with density-dependent host mortality. The stability region specified in Equation (43) for the discrete time, host mortality model (Equations (35) and (36)) as a function of the strength of density-dependent mortality to parasitism $\left(c_{1} / c\right)$ and the number of viable eggs per host, $R$ (blue area). The stability region is smaller with host-feeding, as compared to the density-dependent host mortality model without host-feeding (gray area) (25). Parameters: $\lambda=0, k=1$, and $T=1$.

Singh and Nisbet's (25) model without host-feeding. From this analysis, it is clear that coupling host-feeding with density-dependent host mortality yields a smaller stability region than that of the same model without host-feeding even when ideal conditions for egg load are possible, i.e., $\lambda=0$. Overall, this means the host-feeding dynamic has a destabilizing effect to the non-zero fixed point. We see that when $\lambda=1 / 2$, Equations (35) and (36) reduce to the Singh and Nisbet model (25), but with a scaling of $1 / 2$ on the parasitoid yearly update. In this case, the stability region is exactly halved. As $\lambda \rightarrow 0$, the stability region with host-feeding gets larger but never quite expands to that of the model without host-feeding. This is apparent because $\gamma>1$ for all values of $\lambda$ (when $k=1$ ), which follows from the analysis of the NP fixed point. Therefore, we can conclude that host-feeding is a form of inefficiency 
for the parasitoids that is not ideal for the coexistence of the two species.

\subsection{Density-Dependent Parasitoid Mortality}

Another investigation we considered is a density-dependent parasitoid mortality. Similar to the model presented above, we consider a parasitoid death rate that is dependent on the total parasitoid population. In this sense, our reaction scheme is

$$
\begin{aligned}
& P_{1}+L \stackrel{g(\cdot)}{\longrightarrow} I+P_{0} \\
& P_{0}+L \stackrel{g(\cdot)}{\longrightarrow} P_{1} \\
& P_{0} \stackrel{g_{2}(\cdot)}{\longrightarrow} \text { Death } \\
& P_{1} \stackrel{g_{2}(\cdot)}{\longrightarrow} \text { Death, }
\end{aligned}
$$

where $g_{2}(\cdot)$ is the density-dependent mortality rate of parasitoids. We let $g=c$ as before and define $g_{2}=c_{2}\left(P_{0}+P_{1}\right)$. This model is analogous to Singh and Nisbet's density-dependent parasitoid mortality model without host-feeding (25). As shown by Singh and Nisbet, the non-host-feeding system is stable if $c_{2}>c$. In the host-feeding case, an analytical solution for $I(\tau, t)$ cannot be obtained; however, numerical investigation suggests that the system is stable for $c_{2}$ values that are slightly less than $c$. Indeed, a trajectory with $\lambda=0, k=1, R=2, c=0.01$, and $c_{2}=0.0095$ yields asymptotically stable results whereas the model without host-feeding experiences diverging oscillations. Although the stability region is expanded slightly, we can ultimately conclude that host-feeding has a slight stabilizing effect in the density-dependent parasitoid mortality case.

\section{Quadratic Functional Response with Host-Feeding}

Previous results show that phenomenological update functions with Type II and Type III functional responses do not stabilize the Nicholson-Bailey model $(31 ; 32)$. However, using the semi-discrete framework, Singh et. al. showed that a quadratic functional response yields a neutrally stable fixed point with period $2 \pi / \arctan \left(\sqrt{R^{2}-1}\right)$ in the absence of host-feeding (25). To investigate the effects of host-feeding, we consider a quadratic functional response in the attack rate of Equations (12) and (13) so that $g=c L$. Using Equations 
(14)-(17), our continuous model becomes

$$
\begin{aligned}
\frac{d L}{d \tau} & =-c\left(P_{0}+P_{1}\right) L^{2} \\
\frac{d I}{d \tau} & =c P_{1} L^{2} \\
\frac{d P_{0}}{d \tau} & =c\left(P_{1}-P_{0}\right) L^{2} \\
\frac{d P_{1}}{d \tau} & =-c\left(P_{1}-P_{0}\right) L^{2} .
\end{aligned}
$$

subject to the same initial conditions of Equation (18). In Appendix C, we solve this system explicitly for $L$ and $I$ to obtain the following discrete yearly update system

$$
\begin{aligned}
H_{t+1} & =\frac{R H_{t}}{1+c R H_{t} P_{t}} \\
P_{t+1} & =\frac{k}{2}\left(R H_{t}-H_{t+1}\right)+\left(\frac{1}{2}-\lambda\right) \frac{k P_{t}}{2}\left[1-\exp \left(-\frac{2\left(R H_{t}-H_{t+1}\right)}{P_{t}}\right)\right] .
\end{aligned}
$$

The non-zero fixed point of the system is

$$
H^{*}=\sqrt{\frac{\gamma}{c R T}}, \quad P^{*}=\frac{R-1}{\sqrt{\gamma c R T}},
$$

where $\gamma=\gamma(\lambda, k)$ is defined in Equation (39). Stability analysis (Appendix C) shows that this fixed point is stable for $R>1$ and $\lambda<1 / 2$. Figure 4 illustrates a simulation for $k=1, R=2$ and $c=0.01$ with and without host-feeding. For comparison, we let $\lambda=0$ in the host-feeding model. We can see that without host-feeding, the populations oscillate forever, but with host-feeding, the populations settle to a limiting value. Hence, including host-feeding with a quadratic functional response in both the attack and infection rate stabilized the originally neutrally stable fixed point. It should also be noted that the above host equilibrium is higher than the corresponding quadratic functional response equilibrium in (25) with no host-feeding, by a factor of 1.2344. The parasitoid density is reduced by the same factor. In fact, for any value of $\lambda$, with $k=1$, the host equilibrium is scaled by $\sqrt{\gamma}$ and the parasitoid equilibrium is reduced by the same factor. As we've seen in the previous models, for $\lambda=1 / 2$, this model reduces to the Singh and 

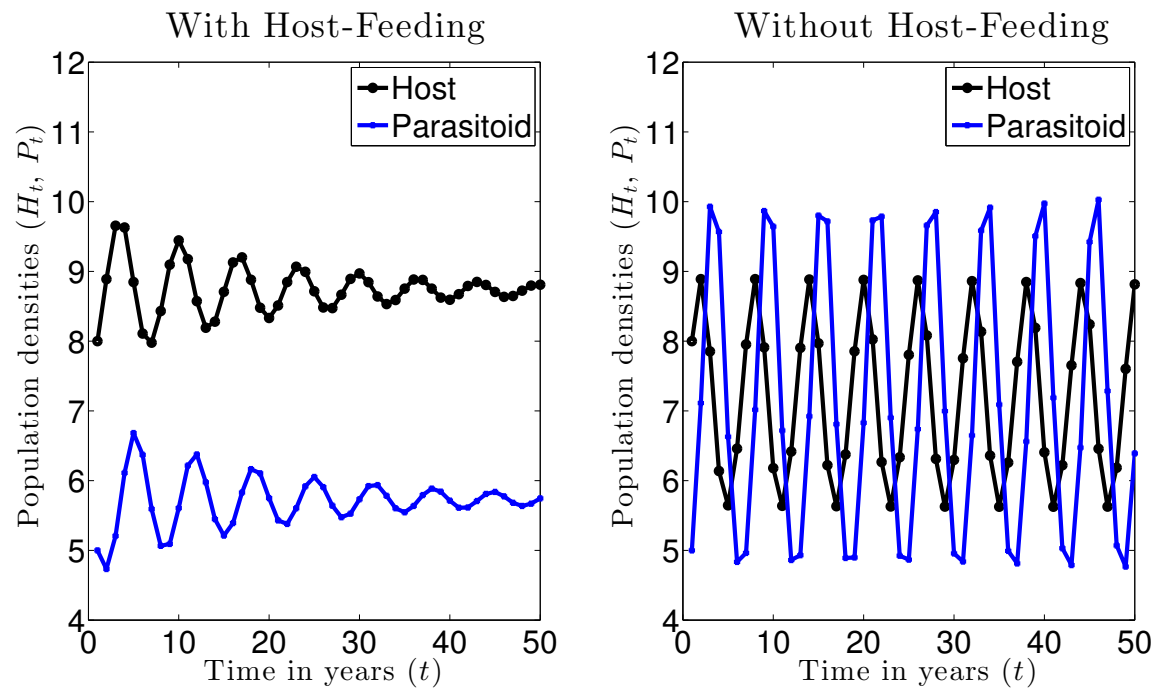

Figure 4: Host-feeding stabilizes the Nicholson-Bailey model with quadratic functional response. Comparison of trajectories of the dynamic interaction given by Equations (49)-(52) (left) to Singh and Nisbet's quadratic functional response model without host-feeding (25) (right). Hostfeeding with a quadratic functional response stabilizes the oscillatory behavior of Singh et. al.'s model. Parameters: $\lambda=0, k=1, T=1, R=2$, and $c=0.01$ with $H_{0}=8$ and $P_{0}=5$.

Nisbet system without host-feeding with a factor of $1 / 2$ in the yearly update for parasitoids (25). Hence, we can conclude that as $\lambda$ increases from 0 to 1 , this model destabilizes, which suggests that the proportion of initially eggless parasitoids determines the fate of both populations. This is clear from the analysis in Appendix C.

\section{Egg Maturation Delay}

The study by Shea et. al. (21) focused on the effects of egg limitation (33) in a host-feeding interaction. They conclude that stability is affected by the length of latent period, i.e., the time it takes for eggs to mature as the gut is emptied. Indeed, the longer the latent period, the longer it takes for the system to stabilize. Among other conclusions, they also observe that stability is not affected by the number of eggs that can be stored. In this sense, our model considers an instantaneous egg maturation process where 
parasitoids carry only a single egg. Because we only consider one egg, we can gather the analytic results studied above. However, to discuss the case of egg maturation, we implement a delay in egg production into the semi-discrete framework with the following reactions

$$
\begin{aligned}
& P_{1}+L \stackrel{g(\cdot)}{\longrightarrow} I+P_{0} \\
& P_{0}+L \stackrel{g(\cdot)}{\longrightarrow} P_{1 / 2} \\
& P_{1 / 2} \stackrel{g_{3}(\cdot)}{\longrightarrow} P_{1} .
\end{aligned}
$$

In contrast to the original model in Section 2 , we add a population, $P_{1 / 2}$, that is analogous to Shea et. al.'s $P_{01}$ population, which represents a parasitoid with zero eggs and a full gut. The new function $g_{3}(\cdot)$ (units: $t i m e^{-1}$ ) represents the maturation rate for a parasitoid to transition from eggless to with egg. We consider the effect of the maturation rate on the stable system of Section 5. In the simplest case, we assume the maturation delay is constant, $g_{3}=c_{3}$, and we let $g=c L$ as before. Using these reactions with the initial conditions of Equation (18) and $P_{1 / 2}(0, t)=0$, our equations become

$$
\begin{aligned}
\frac{d L}{d \tau} & =-c\left(P_{0}+P_{1}\right) L^{2} \\
\frac{d I}{d \tau} & =c P_{1} L^{2} \\
\frac{d P_{0}}{d \tau} & =c\left(P_{1}-P_{0}\right) L^{2} \\
\frac{d P_{1 / 2}}{d \tau} & =c P_{0} L^{2}-c_{3} P_{1 / 2} \\
\frac{d P_{1}}{d \tau} & =c_{3} P_{1 / 2}-c P_{1} L^{2} .
\end{aligned}
$$

Analytical solutions to this model are unattainable, but we confirm the destabilizing effect of the latent period numerically. In Figure 5, we see a series of host densities using the quadratic functional response model plotted against time with a varying delay parameter, $1 / c_{3}$ (plotted as $\log \left(1 / c_{3}\right)$ ). As the rate of maturation gets slower, the delay increases. For a critical delay the stable host-feeding, functional response model becomes unstable as the density of hosts begins to experience diverging oscillations.

It is worthwhile to note that the characteristic time scale, $T$, in which the vulnerable period takes place is approximately 90 days. The instability 


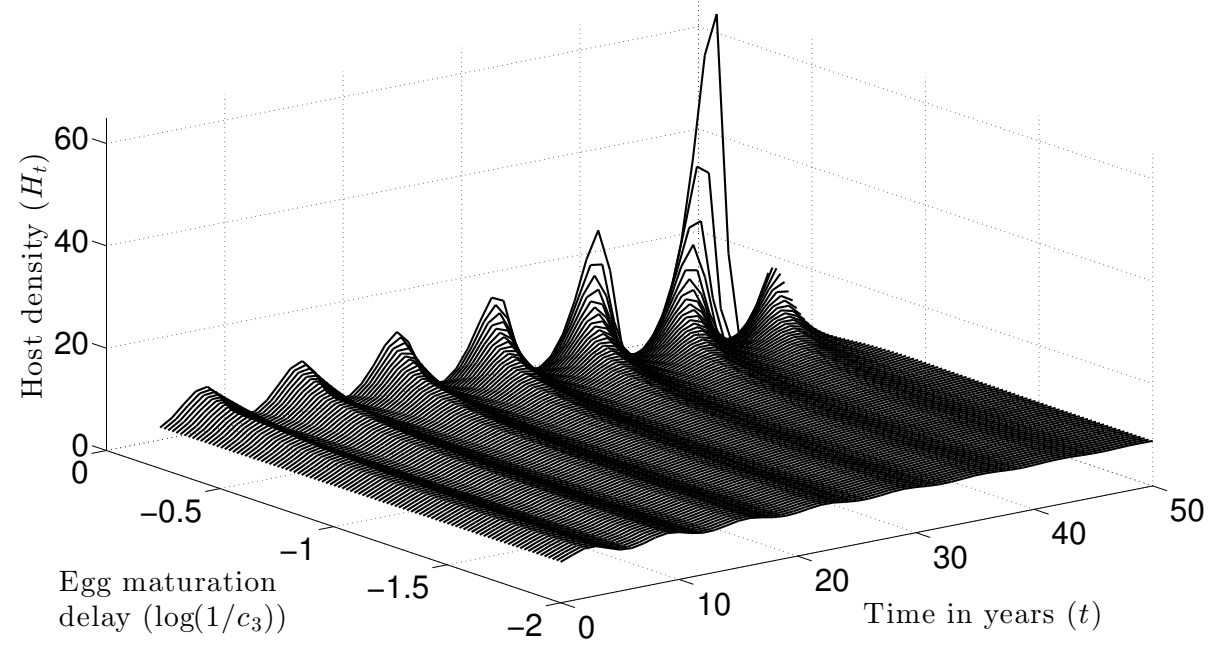

Figure 5: Incorporating a delay due to egg production has a destabilizing effect on the quadratic functional response model. A series of host population density trajectories $\left(H_{t}\right.$ on the $z$-axis $)$ are plotted against time ( $t$ on the $x$-axis) for each value of egg maturation delay $\left(\log \left(1 / c_{3}\right)\right)$ on the $y$-axis). As the delay time, $1 / c_{3}$, approaches 0.33 or approximately 30 days, the system becomes unstable. However, the system is always stable for smaller (on the order of hours) delay time. Parameters: $\lambda=0, k=1, T=1$, $R=2$, and $c=0.01$ with $H_{0}=8$ and $P_{0}=5$.

is caused by a delay that is approximately 0.33 , which suggests that the egg maturation delay is approximately 30 days, which is far too long for an egg to mature. As illustrated in Figure 5, a delay value closer to $10^{-2}$ or $10^{-3}$, which means it takes on the order of hours for an egg to mature, yields stable results. Therefore, in this more realistic case, the system is always stable.

\section{Discussion}

In this paper, we have considered the classic host-parasitoid interaction with a host-feeding dynamic. For simplicity and to obtain analytical results, our model ignores gut capacity and includes two states, eggless and with egg, where eggs are produced immediately after host consumption. In contrast to previous phenomenological models, we incorporate the semi-discrete 
framework, which has more relevance to parasitoid populations with one year life cycles and allows us to track the change in hosts during the vulnerable period. The preceding analyses show that the effects of host-feeding alone cannot stabilize the classic Nicholson-Bailey model. However, including density-dependence or a quadratic functional response with the host-feeding dynamic can provide either a destabilizing or stabilizing effect as compared to Singh and Nisbet's results (25).

Coupling host-feeding with a density-dependent host mortality creates a smaller parameter space that allows for a stable host-parasitoid equilibrium. For example, when $R=2$ and $c=1$ a stable host-parasitoid fixed point exists in a smaller range of density-dependent mortality rates by a factor of 1.4184 so that for higher values of density-dependent mortality, stability no longer occurs. Indeed, the maximum host mortality rate allowing for a stable host-parasitoid equilibrium is $c_{1}=0.4549$ with host-feeding and without host-feeding, the maximum mortality rate is $c_{1}=0.6931$. This means host-feeding enhances the effect of density-dependent mortality. Therefore, without host-feeding, higher rates of mortality exist in the stable interaction. On the other hand, if we increase $c_{1}$ from from a lower level, we find that the host-feeding model stabilizes first. However, the difference in the lower boundaries of both models is smaller, making the stability region narrower with host-feeding. Also, with host-feeding, the mean host density is increased by a factor of 1.5412 in the host-parasitoid stability region, while the mean parasitoid density decreases by a factor of 3.5319. It is apparent that host-feeding makes the parasitoid more efficient in reducing the number of hosts in every generation; however, it reduces the number of potential hosts available for oviposition. This justifies the expanded no-parasitoid stability region which suggests that host-feeding is costly for parasitoid reproduction each year. Host-feeding may have a short term biological control effect but ultimately it reduces the parasitoid population in the long term.

Host-feeding has a stabilizing effect when coupled with a quadratic functional response or a density-dependent parasitoid mortality. In the case of a quadratic functional response in the attack rates, Singh and Nisbet show that the sole equilibrium point is neutrally stable (25). In our model, host-feeding stabilizes this equilibrium point and also shows an increase in the mean host density by a factor of 1.2344. The mean parasitoid density is reduced by the same factor. In this model, the parasitism and host-feeding rate is dependent on the host density, and since host-feeding reduces the amount of host larvae, host-feeding relaxes the effect of parasitism and down-regulates 
itself. The result of this is a stable host-parasitoid equilibrium. In the case when there is a density-dependent parasitoid mortality, numerical evidence suggests that host-feeding expands the stability region slightly. This suggests that host-feeding counteracts the parasitoid reduction by decreasing the host density. This allows a slight relaxation in the stable parameter for parasitoid mortality as compared to parasitism and host-feeding. Hence, we may conclude that host-feeding brings stability to both systems because it effectively eliminates the host population during the vulnerable period, but this in turn decreases the efficiency of the parasitoid to reproduce from year to year.

Investigating other key assumptions in our model leads to further insight into the host-feeding dynamic. For instance, for most comparisons we assume parasitoids emerge into the vulnerable period each year with an egg. As Jervis and Harvey explain in (34), the adult parasitoid female emerges with eggs, and later feeds on hosts to gain further eggs. However, do all females enter the vulnerable period with eggs? Dieckhoff et. al. and others conclude that many psychological and behavioral factors including resorption and nutrition may contribute to the specific egg load at any given time (28). This translates to the initial condition of our continuous model. We investigate this by considering a proportion of the starting population that is initially eggless, i.e., $P_{0}(0)=\lambda P_{t}$ and $P_{1}(0)=(1-\lambda) P_{t}$. We found that as $\lambda \rightarrow 1$ (poor conditions for full egg load at adult emergence), every model becomes less stable. Indeed, for $\lambda=1 / 2$, it is clear that Equations (23)-(24), Equations (35)-(36), and Equations (53)-(54) all yield an identical system to Singh and Nisbet's semi-discrete models without host-feeding, except that there is a factor of $1 / 2$ on the parasitoid yearly update. In the host-mortality model of Section 4.1, we note that if conditions for egg load are ideal $(\lambda=0)$, the scaling factor $\gamma$ does not achieve a value of 2 , which would effectively match the same model without host-feeding. Instead, we find that $\gamma=1.5328$ suggesting that the yearly initial proportion of eggless parasitoids creates a burden on the host-parasitoid equilibrium state. Similarly, in the quadratic functional response model of Section 5, we find that stability only occurs if $\lambda<1 / 2$. In general, we conclude that the initial proportion of eggless parasitoids at the start of the vulnerable period each year has a long term effect on both populations.

Finally, we note that many other important consumer-resource dynamics can be easily implemented into the semi-discrete framework. For example, we confirmed that an egg maturation delay produces an instability when coupled with the host-feeding dynamic by considering a simple transition reaction 
from eggless to with egg parasitoids. A necessary future step would be to investigate the effects of a larger egg load and/or to implement a probability that a parasitoid will host feed. In this sense, an optimal stability criterion could exist. Furthermore, we seek to investigate susceptibility of host risk to parasitoid attack, as in (27). Susceptibility of risk cannot stabilize the model with host-feeding alone, but could provide interesting results with host-dependent mortality or a quadratic functional response. The generality of the semi-discrete framework allows us to implement these changes with ease.

\section{Acknowledgements}

The authors would like to thank Bill Murdoch and Roger Nisbet for their helpful discussions. Brooks Emerick would like to thank his advisor, Dr. Gilberto Schleiniger, for his continued support, and Zhenyu He and Longfei Li for helpful discussions. Also, A. Singh would like to acknowledge the support from the National Science Foundation Grant DMS-1312926, University of Delaware Research Foundation (UDRF) and Oak Ridge Associated Universities (ORAU).

\section{Appendix A. Analysis of Nicholson-Bailey model with host-feeding}

We consider the explicit solution to Equations (19)-(22) using the initial conditions of Equation (18). Adding Equations (21) and (22) gives

$$
\frac{d P_{0}}{d \tau}+\frac{d P_{1}}{d \tau}=0 \quad \Rightarrow \quad P_{0}+P_{1}=P_{t}
$$

Substituting $P_{1}=P_{t}-P_{0}$ into Equation (19) yields

$$
\frac{d L}{d \tau}=-c P_{t} L \quad \Rightarrow \quad L(\tau, t)=R H_{t} e^{-c P_{t} \tau} .
$$

We can now solve for $P_{0}$ by substituting our expression for $L$ and $P_{1}$ into Equation (21) to obtain

$$
\frac{d P_{0}}{d \tau}+2 c R H_{t} e^{-c P_{t} \tau} P_{0}=c R H_{t} P_{t} e^{-c P_{t} \tau} .
$$


Using the integrating factor to solve this equation, we get an expression for both $P_{0}$ and $P_{1}$. We have

$$
\begin{aligned}
& P_{0}(\tau, t)=\frac{P_{t}}{2}+\left(\lambda-\frac{1}{2}\right) P_{t} \exp \left[\frac{R H_{t}}{P_{t}}\left(1-e^{-c P_{t} \tau}\right)\right] \\
& P_{1}(\tau, t)=\frac{P_{t}}{2}+\left(\frac{1}{2}-\lambda\right) P_{t} \exp \left[\frac{R H_{t}}{P_{t}}\left(1-e^{-c P_{t} \tau}\right)\right] .
\end{aligned}
$$

Finally, substituting our expression for $P_{1}$ and $L$ into Equation (20) gives

$$
\frac{d I}{d \tau}=\frac{c R H_{t} P_{t}}{2} e^{-c P_{t} \tau}+\left(\frac{1}{2}-\lambda\right) c R H_{t} P_{t} e^{-c P_{t} \tau} \exp \left[\frac{R H_{t}}{P_{t}}\left(1-e^{-c P_{t} \tau}\right)\right] .
$$

Solving this and applying the initial condition gives the following expression for $I(\tau, t)$

$$
I(\tau, t)=\frac{R H_{t}}{2}\left(1-e^{-c P_{t} \tau}\right)+\left(\frac{1}{2}-\lambda\right) P_{t}\left\{\exp \left[\frac{R H_{t}}{P_{t}}\left(1-e^{-c P_{t} \tau}\right)\right]-1\right\} .
$$

Using the solutions for $L$ and $I$, we find the update as in Equations (23) and (24) using the definition in Equations (8) and (9). We can analyze the resulting discrete-time model by implementing the procedure outlined in (26). Using a general discrete model such as

$$
\begin{aligned}
H_{t+1} & =F\left(H_{t}, P_{t}\right) \\
P_{t+1} & =G\left(H_{t}, P_{t}\right),
\end{aligned}
$$

we can perform a linear stability analysis about the fixed point $\left(H^{*}, P^{*}\right)$, where $H^{*}$ and $P^{*}$ satisfy the following system of equations

$$
\begin{aligned}
H^{*} & =F\left(H^{*}, P^{*}\right) \\
P^{*} & =G\left(H^{*}, P^{*}\right),
\end{aligned}
$$

by determining if the spectral radius of the Jacobian matrix evaluated at the fixed point is less than one. That is, if the magnitude of the eigenvalues of the following Jacobian matrix,

$$
J:=J\left(H^{*}, P^{*}\right)=\left[\begin{array}{ll}
\left.\frac{\partial F}{\partial H}\right|_{\left(H^{*}, P^{*}\right)} & \left.\frac{\partial F}{\partial P}\right|_{\left(H^{*}, P^{*}\right)} \\
\left.\frac{\partial G}{\partial H}\right|_{\left(H^{*}, P^{*}\right)} & \left.\frac{\partial G}{\partial P}\right|_{\left(H^{*}, P^{*}\right)}
\end{array}\right],
$$


are within the unit circle, then the fixed point $\left(H^{*}, P^{*}\right)$ is asymptotically stable. The eigenvalues fall within the unit circle if the following three Jury conditions hold,

$$
\begin{aligned}
1-\operatorname{Tr}(J)+\operatorname{Det}(J) & >0 \\
1+\operatorname{Tr}(J)+\operatorname{Det}(J) & >0 \\
1-\operatorname{Det}(J) & >0 .
\end{aligned}
$$

In the case of the Nicholson-Bailey model with host-feeding, our update functions are given by Equations (23) and (24),

$$
\begin{aligned}
F\left(H_{t}, P_{t}\right) & =R H_{t} e^{-c P_{t} T} \\
G\left(H_{t}, P_{t}\right)=\frac{k R H_{t}}{2} & \left(1-e^{-c P_{t} T}\right)+\cdots \\
& \left(\frac{1}{2}-\lambda\right) k P_{t}\left\{\exp \left[\frac{R H_{t}}{P_{t}}\left(1-e^{-c P_{t} T}\right)\right]-1\right\},
\end{aligned}
$$

with fixed point

$$
H^{*}=\frac{\beta \ln R}{c T(R-1)}, \quad P^{*}=\frac{\ln R}{c T},
$$

where $\beta=\beta(\lambda, k)$ is given by the function

$$
\beta(\lambda, k)=1-2 \lambda+\frac{2}{k}-\text { LambertW }\left[(1-2 \lambda) \exp \left(1-2 \lambda+\frac{2}{k}\right)\right] .
$$

We note that the function $\beta$ becomes imaginary as $\lambda$ increases from 0 to 1 . Also, as $k \rightarrow \infty$, we find that $\beta \rightarrow 0$, which implies that as the number of parasitoid larvae increases, the equilibrium host population tends to zero. In any case, we can evaluate the trace and determinant of the Jacobian matrix as

$$
\begin{aligned}
\operatorname{Tr}(J) & =1+\frac{k}{2} \frac{\beta \ln R}{R-1}+k\left(\lambda-\frac{1}{2}\right)\left[1-(1-\beta) e^{\beta}-\frac{\beta \ln R}{R-1}\right] \\
\operatorname{Det}(J) & =\frac{k}{2} \frac{\beta(2-R) \ln R}{R-1}+k\left(\lambda-\frac{1}{2}\right)\left[1-(1-\beta) e^{\beta}-\frac{\beta(2-R) \ln R}{R-1}\right] .
\end{aligned}
$$

We find that $1-\operatorname{Tr}(J)+\operatorname{Det}(J)<0$ for $R>1$ for any real value of $\beta$. Hence, the fixed point is unstable. 


\section{Appendix B. Analysis of density-dependent host mortality model}

We consider the explicit solution to Equations (31)-(34). Adding Equations (33) and (34) gives $P_{0}+P_{1}=P_{t}$, which upon substitution of $P_{1}=P_{t}-P_{0}$ into Equation (31) yields

$$
\frac{d L}{d \tau}=\left(-c P_{t}+c_{1} L\right) L \quad \Rightarrow \quad L(\tau, t)=\frac{R H_{t} e^{-c P_{t} \tau}}{1+c_{1} R H_{t} \frac{1-e^{-c P_{t} \tau}}{c P_{t}}} .
$$

The previous differential equation can be solved using partial fraction decomposition. We can now solve for $P_{0}$ by substituting our expression for $L$ and $P_{1}$ into Equation (33) to obtain

$$
\frac{d P_{0}}{d \tau}+\frac{2 c R H_{t} e^{-c P_{t} \tau}}{1+c_{1} R H_{t} \frac{1-e^{-c P_{t} \tau}}{c P_{t}}} P_{0}=\frac{c R H_{t} P_{t} e^{-c P_{t} \tau}}{1+c_{1} R H_{t} \frac{1-e^{-c P_{t} \tau}}{c P_{t}}} .
$$

Using the integrating factor to solve this equation, we get an expression for both $P_{0}$ and $P_{1}$. Solving this explicitly for $P_{0}$ and using the initial condition from the previous section yields our solution for both $P_{0}$ and $P_{1}$ as

$$
\begin{aligned}
& P_{0}(\tau, t)=\frac{P_{t}}{2}+\frac{\left(\lambda-\frac{1}{2}\right) P_{t}}{\left(1+c_{1} R H_{t} \frac{1-e^{-c P_{t} \tau}}{c P_{t}}\right)^{\frac{2 c}{c_{1}}}} \\
& P_{1}(\tau, t)=\frac{P_{t}}{2}+\frac{\left(\frac{1}{2}-\lambda\right) P_{t}}{\left(1+c_{1} R H_{t} \frac{1-e^{-c P_{t} \tau}}{c P_{t}}\right)^{\frac{2 c}{c_{1}}}} .
\end{aligned}
$$

Finally, substituting our expression for $P_{1}$ into Equation (32) gives

$$
\frac{d I}{d \tau}=\frac{c R H_{t} P_{t} e^{-c P_{t} \tau}}{2\left(1+c_{1} R H_{t} \frac{1-e^{-c P_{t} \tau}}{c P_{t}}\right)}+\frac{\left(\lambda-\frac{1}{2}\right) c R H_{t} P_{t} e^{-c P_{t} \tau}}{\left(1+c_{1} R H_{t} \frac{1-e^{-c P_{t} \tau}}{c P_{t}}\right)^{\frac{2 c}{c_{1}}+1}} .
$$

Solving this and applying the initial condition gives the following expression for $I(\tau, t)$

$$
\begin{aligned}
I(\tau, t)=\frac{c P_{t}}{2 c_{1}} \ln & \left(1+c_{1} R H_{t} \frac{1-e^{-c P_{t} \tau}}{c P_{t}}\right)+\cdots \\
& \left(\lambda-\frac{1}{2}\right) \frac{P_{t}}{2}\left[\left(1+c_{1} R H_{t} \frac{1-e^{-c P_{t} \tau}}{c P_{t}}\right)^{-\frac{2 c}{c_{1}}}-1\right] .
\end{aligned}
$$


Using the solutions for $L$ and $I$, we find the update as in Equations (35) and (36) using the definition in Equations (8) and (9). In the density-dependent host mortality case, our discrete update functions are,

$$
\begin{aligned}
H_{t+1} & =\frac{R H_{t} e^{-c P_{t} T}}{f\left(H_{t}, P_{t}\right)} \\
P_{t+1} & =\frac{c k P_{t}}{2 c_{1}} \ln \left[f\left(H_{t}, P_{t}\right)\right]+\left(\lambda-\frac{1}{2}\right) \frac{k P_{t}}{2}\left[f\left(H_{t}, P_{t}\right)^{-\frac{2 c}{c_{1}}}-1\right]
\end{aligned}
$$

where

$$
f\left(H_{t}, P_{t}\right)=1+c_{1} R H_{t} \frac{1-e^{-c P_{t} T}}{c P_{t}} .
$$

This system has two nontrivial fixed points. As noted in the main text, the first no-parasitoid equilibrium point is given by

$$
H_{N P}^{*}=\frac{R-1}{c_{1} T R}, \quad P^{*}=0 .
$$

Denoting the Jacobian matrix for the first fixed point by $J_{N P}$, we find the trace and determinant to be

$$
\begin{aligned}
\operatorname{Tr}\left(J_{N P}\right) & =\frac{1}{R}-\frac{k}{2}\left[\left(\frac{1}{2}-\lambda\right)\left(R^{-\frac{2}{z}}-1\right)-\frac{\ln R}{z}\right] \\
\operatorname{Det}\left(J_{N P}\right) & =-\frac{k}{2 R}\left[\left(\frac{1}{2}-\lambda\right)\left(R^{-\frac{2}{z}}-1\right)-\frac{\ln R}{z}\right],
\end{aligned}
$$

where $z=c_{1} / c$. Using these expressions, Jury condition 1 (Equation (A.13)) gives the most strict condition so that $z>\ln R / \gamma$ for stability, where $\gamma=$ $\gamma(\lambda, k)$ is defined as

$$
\gamma(\lambda, k)=\lambda-\frac{1}{2}+\frac{2}{k}+\frac{1}{2} \text { LambertW }\left[(1-2 \lambda) \exp \left(1-2 \lambda-\frac{4}{k}\right)\right] .
$$

Here, $\gamma$ is a real-valued function for all $\lambda$ and $k$ and is maximized when $\lambda=1$ and $k=1$. Figure 2 refers to the inequality $z>\ln R / \gamma$ for $k=1$ and $\lambda=0,1 / 2,1$. The second equilibrium point is given by

$$
H^{*}=\left(\frac{\exp \left(\frac{\gamma c_{1}}{c}\right)-1}{R-\exp \left(\frac{\gamma c_{1}}{c}\right)}\right) \frac{c P^{*}}{c_{1}}, \quad P^{*}=\frac{\ln (R)-\frac{\gamma c_{1}}{c}}{c T},
$$


where $\gamma=\gamma(\lambda, k)$ is defined by Equation (B.13). Define $J$ as the Jacobian matrix evaluated at the second equilibrium point above, then the trace and determinant are given by

$$
\begin{aligned}
& \operatorname{Tr}(J)=\frac{k}{2}\left[\frac{1-\gamma z+\ln R-R e^{-\gamma z}}{z\left(R-e^{\gamma z}\right)}\right]\left[1-(2 \lambda-1) e^{-2 \gamma}\right]\left(e^{\gamma z}-1\right)+\cdots \\
& \operatorname{Det}(J)=\frac{k}{2}\left[\frac{R e^{-\gamma z}(\ln R-\gamma z-1)+1}{z\left(R-e^{\gamma z}\right)}\right]\left[1-(2 \lambda-1) e^{-2 \gamma}\right]\left(e^{\gamma z}-1\right)+\cdots \\
& \frac{k}{2}\left[\gamma+\left(\lambda-\frac{1}{2}\right)\left(e^{-2 \gamma}-1\right)\right] e^{-\gamma z}
\end{aligned}
$$

Here, Jury condition 3 is the most strict, and we must have $z^{*}<z<\ln R / \gamma$, where $z^{*}$ solves $1-\operatorname{Det}(J)=0$. Equation (44) refers to Jury condition 3 with $\lambda=0$ and $k=1$. Figure 3 shows a plot for the stability region without host-feeding $(\lambda=1 / 2$ and $k=2)$ and with host-feeding $(\lambda=0$ and $k=1)$.

\section{Appendix C. Analysis of quadratic functional response model}

We consider the explicit solution to Equations (49)-(52). Adding Equations (49) and (52) gives $P_{0}+P_{1}=P_{t}$. Substituting $P_{1}$ into Equation (49) yields

$$
\frac{d L}{d \tau}=-c P_{t} L^{2} \quad \Rightarrow \quad L(\tau, t)=\frac{R H_{t}}{1+c R H_{t} P_{t} \tau} .
$$

We can now solve for $P_{0}$ by substituting our expression for $L$ and $P_{1}$ into Equation (49) to obtain

$$
\frac{d P_{0}}{d \tau}+\frac{2 c R^{2} H_{t}^{2}}{\left(1+c R H_{t} P_{t} \tau\right)^{2}} P_{0}=\frac{c R^{2} H_{t}^{2} P_{t}}{\left(1+c R H_{t} P_{t} \tau\right)^{2}}
$$

Using the integrating factor to solve this equation, we get an expression for both $P_{0}$ and $P_{1}$. We have

$$
\begin{aligned}
& P_{0}(\tau, t)=\frac{P_{t}}{2}+\left(\lambda-\frac{1}{2}\right) P_{t} \exp \left[\frac{2 R H_{t}}{P_{t}}\left(\frac{1}{1+c R H_{t} P_{t} \tau}-1\right)\right] \\
& P_{1}(\tau, t)=\frac{P_{t}}{2}+\left(\frac{1}{2}-\lambda\right) P_{t} \exp \left[\frac{2 R H_{t}}{P_{t}}\left(\frac{1}{1+c R H_{t} P_{t} \tau}-1\right)\right] .
\end{aligned}
$$


Substituting our expressions for $P_{1}$ and $L$ into Equation (50), we obtain the following ODE for $I$,

$$
\frac{d I}{d \tau}=\frac{c R^{2} H_{t}^{2} P_{t}}{2\left(1+c R H_{t} P_{t} \tau\right)^{2}}+\left(\frac{1}{2}-\lambda\right) \frac{c R^{2} H_{t}^{2} P_{t} \exp \left[\frac{2 R H_{t}}{P_{t}}\left(\frac{1}{1+c R H_{t} P_{t} \tau}-1\right)\right]}{2\left(1+c R H_{t} P_{t} \tau\right)^{2}} .
$$

We can solve this equation directly to obtain the following expression for $I$,

$$
\begin{aligned}
I(\tau, t)=\frac{R H_{t}}{2} & \left(1-\frac{1}{1+c R H_{t} P_{t} \tau}\right)+\cdots \\
& \left(\frac{1}{2}-\lambda\right) \frac{P_{t}}{2}\left\{1-\exp \left[\frac{2 R H_{t}}{P_{t}}\left(\frac{1}{1+c R H_{t} P_{t} \tau}-1\right)\right]\right\} .
\end{aligned}
$$

Using the solutions for $L$ and $I$, we find the update as in Equations (53) and (54) using the definition in Equations (8) and (9). In the quadratic functional response case, our discrete update functions are,

$$
\begin{aligned}
F\left(H_{t}, P_{t}\right)= & \frac{R H_{t}}{1+c R H_{t} P_{t} T} \\
G\left(H_{t}, P_{t}\right)= & \frac{k R H_{t}}{2}\left(1-\frac{1}{1+c R H_{t} P_{t} T}\right)+\cdots \\
& \left(\frac{1}{2}-\lambda\right) \frac{k P_{t}}{2}\left\{1-\exp \left[\frac{2 R H_{t}}{P_{t}}\left(\frac{1}{1+c R H_{t} P_{t} T}-1\right)\right]\right\},
\end{aligned}
$$

which gives rise to the following fixed point

$$
H^{*}=\sqrt{\frac{\gamma}{c R T}}, \quad P^{*}=\frac{R-1}{\sqrt{\gamma c R T}},
$$

where $\gamma=\gamma(\lambda, k)$ is defined in Equation (B.13). The trace and determinant of the Jacobian matrix is given by

$$
\begin{gathered}
\operatorname{Tr}(J)=k\left(\lambda-\frac{1}{2}\right)\left[\left(\gamma+\frac{1}{2}\right) e^{-2 \gamma}-\frac{1}{2}\right]+\cdots \\
\frac{1}{R}\left\{1+\frac{\gamma k}{2}\left[1-(2 \lambda-1) e^{-2 \gamma}\right]\right\} \\
\operatorname{Det}(J)=\frac{\gamma k}{2}\left[1-(2 \lambda-1) e^{-2 \gamma}\right]+\left(\lambda-\frac{1}{2}\right) \frac{k}{R}\left[\left(\gamma+\frac{1}{2}\right) e^{-2 \gamma}-\frac{1}{2}\right] .
\end{gathered}
$$


All three Jury conditions hold for $R>1$ and $\lambda<1 / 2$.

\section{References}

[1] B. A. Hawkins. Parasitoid Community Ecology. Oxford University Press, New York, NY, 1994.

[2] B. A. Hawkins. Patterns and Process in Host-Parasitoid Interactions. Cambridge University Press, New York, NY, 1994.

[3] M. A. Jervis and N. A. C. Kidd. Host-feeding strategies in hymenopteran parasitoids. Biol Rev, 61(4):395-434, 1986.

[4] N. A. C. Kidd and M. A. Jeris. The effects of host-feeding behaviour on the dynamics of parasitoid-host interactions, and the implications for biological control. Res Popul Ecol, 31(2):2435-274, 1989.

[5] P. De Bach. The importance of host-feeding by adult parasites in the reduction of host populations. J Econ Entomol, 36(5):647-653, 1943.

[6] S. E. Flanders. Predatism by the adult hymenopterous parasite and its role in biological control. J Econ Entomol, 46(4):541-544, 1953.

[7] M. A. Jervis, B. A. Hawkin, and N. A. C. Kidd. The usefulness of destructive host-feeding parasitoids in classical biological control: theory and observation conflict. Ecological Entomology, 21(1):41-46, 1996.

[8] T. Ueno. Selective host-feeding on parasitized hosts by the parasitoid itoplectis naranyae (hymenoptera: Ichneumonidae) and its implication for biological control. Bullletin of Entomological Research, 88(4):461466, 1998.

[9] A. Nicholson and V. A. Bailey. The balance of animal populations. part 1. Prc Zool Soc London, 105(3):551-598, 1935.

[10] A. Bompard, I. Amat, X. Fauvergue, and T. Spataro. Host-parasitoid dynamcis and the success of biological control when parasitoids are prone to allele effects. Plos Biol, 8(10):233-253, 2013.

[11] S. R. Jang and J. L. Yu. Discrete-time host-parasitoid models with pest control. J Biol Dyn, 6(2):718-739, 2012. 
[12] E. Hackett-Jones, A. White, and C. A. Cobbold. The evolution of developmental timing in natural enemy systems. J Theor Biol, 275(1):1-11, 2011.

[13] C. A. Cobbold, J. Roland, and M. A. Lewis. The impact of parasitoid emergence time on host-parastioid population dynamics. Theor Popul Biol, 75(2):201-215, 2009.

[14] S. Kapcak, U. Ufuktepe, and S. Elaydi. Stability and invariant manifolds of a generalized beddington host-parasitoid model. J Theor Biol, 7(1):233-253, 2013.

[15] W. W. Murdoch, C. J. Briggs, and R. M. Nisbet. Consumer-Resource Dynamics. Princeton University Press, Princeton, NJ, 2003.

[16] N. Yamamura and E. Yano. A simple model of host-parasitoid interaction with host-feeding. Res Popul Ecol, 30(2):353-369, 1988.

[17] W. W. Murdoch, R. M. Nisbet, R. F. Luck, H. C. J. Godfray, and W. S. C. Gurney. Size-selective sex-allocation and host-feeding in a parasitoid-host model. J Anim Ecol, 61(3):533-541, 1992.

[18] C. J. Briggs, R. M. Nisbet, W. W. Murdoch, T. R. Collier, and J. Metz. Dynamical effects of host-feeding. J Anim Ecol, 64(3):403-416, 1995.

[19] N. A. C. Kidd and M. A. Jervis. Host-feeding and oviposition strategies of parasitoids in relation to host stage. Res Popul Ecol, 33(1):13-28, 1991a.

[20] N. A. C. Kidd and M. A. Jervis. Host-feeding and oviposition strategies of parasitoids in relation to host stage: consequences for parasitoid-host population dynamics. Res Popul Ecol, 33(1):87-99, 1991b.

[21] K. Shea, R. M. Nisbet, W. W. Murdoch, and H. J. S. Yoo. The effect of egg limitation on stability in insect host-parasitoid population models. J Anim Ecol, 65(6):743-755, 1996.

[22] M. P. Hassell. Host-parasitoid population dynamics. J Anim Ecol, 69(4):543-566, 2000.

[23] M. P. Hassell. The Spatial and Temporal Dynamics of Host Parasitoid Interactions. Oxford University Press, New York, NY, 2000. 
[24] E. Pachepsky, R. M. Nisbet, and W. W. Murdoch. Between discrete and continuous: Consumer-resource dynamics with synchronized reproduction. Ecology, 89(1):280-288, 2007.

[25] A. Singh and R. M. Nisbet. Semi-discrete host-parasitoid models. J Theor Biol, 247(4):733-742, 2007.

[26] S. Elaydi. An Introduction to Difference Equations. Springer, New York, NY, 1996.

[27] A. Singh, W. W. Murdoch, and R. M. Nisbet. Skewed attacks, stability, and host suppression. Ecology, 90(6):1679-1686, 2009.

[28] C. Dieckhoff and G. E. Heimpel. Determinants of egg load in the soybean aphid parasitoid binodoxys communis. Entomologia Experimentalis et Applicata, 136(3):254-261, 2010.

[29] R. M. May, M. P. Hassell, R. M. Anderson, and D. W. Tonkyn. Density dependence in host-parasitoid models. J Anim Ecol, 50(3):855-865, 1981.

[30] M. P. Hassell and G. C. Varley. New inductive population model for insect and its bearing on biological control. Nature, 223(1):1133-1137, 1969.

[31] D. J. Rogers. Random searching and incest population models. J Anim Ecol, 41(1):369-383, 1972.

[32] M. P. Hassell and H. N. Comins. Sigmoid functional responses and population stability. Theor Popul Biol, 14(1):62-66, 1978.

[33] J. D. Reeve and W. W. Murdoch. Aggregation by parasitoids in the successful control of the california red scale: a test of theory. J Anim Ecol, 54(3):797-816, 1985.

[34] M. A. Jervis and J. A. Harvey. Rosource acquisition, allocation, and utilization in parasitoid reproductive strategies. Annu Rev Entomol, 53(1):361-385, 2008. 